\title{
EEG correlates of posthypnotically controlled degrees of cognitive arousal
}

\author{
GERALD S. BLUM and JOHN K. NASH \\ University of California, Santa Barbara, California 93106
}

\begin{abstract}
Experimental control over five degrees of cognitive (as opposed to organismic) arousal has been developed by hypnotic programming techniques. Previously, these posthypnotic manipulations have been applied to the investigation of diverse topics such as visual discrimination, performance on the Stroop test, selective concentration on color ve. form of consonants, and cognitive "reverberation." The present study explored electroencephalographic (EEG) correlates of the five degrees of cognitive arousal in a task requiring participants to visualize objects for 1-min periods while lying on a couch with their eyes closed. Analysis of data from the occipital area in left and right hemispheres revealed that the highest degree of arousal was accompanied by larger amplitudes of alpha and beta power and smaller amplitudes of theta. This pattern of results was similar in both hemispheres, although more marked in the left. The findings, which provide an independent source of support for validity of the hypnotic programming, are discussed in relation to EEG literature on cognitive activity.
\end{abstract}

This investigation was undertaken to provide independent electroencephalographic (EEG) evidence bearing on the validity of posthypnotically controlled degrees of cognitive arousal. In the past, a conceptual distinction between "cognitive" and "organismic" arousal was drawn to separate mental from somatic components of arousal, and a hypnotic programming technique was first used to operationalize the distinction in the context of a visual discrimination task (Blum, Geiwitz, \& Stewart, 1967). In the course of their hypnotic training, participants learn to regulate their mental alertness and concentration along a 5-point scale ranging from a peak at which mental contents are exceptionally vivid and clear (labeled "+AA") down to a nearly stuporous state in which mental contents are extremely vague and fuzzy ("-AA"); the midpoint ("0") corresponds to a normal waking level of alertness, and the halfway points between 0 and the two extremes are labeled "+A" and "-A." Somatic manifestations of arousal, such as changes in muscle tension, heart rate, and skin resistance, are specifically excluded in the training. Once learned under hypnosis, the cues are administered posthypnotically with amnesia for the prior training. Details of these hypnotic procedures are presented elsewhere (Blum, 1979).

Degrees of cognitive arousal, induced by this method, have been shown to affect a wide range of dependent variables including, in the study noted above (Blum,

This research was supported in part by a general research grant from the University of California, Santa Barbara. We wish to express our appreciation to John F. Kihlstrom and Robert A. Bjork for their editorial assistance and to William Schramm, Master of Scientific Instrumentation, for his design and construction of the EEG signal analyzer. Requests for reprints should be sent to Gerald S. Blum, Department of Psychology, University of California, Santa Barbara, California 93106.
Geiwitz, \& Stewart, 1967), response time for the identification of tachistoscopically flashed Xs varying in dark. ness of line, performance on the Stroop color-word test (Blum \& Graef, 1971), and the capacity for selective concentration on color vs. form of consonants (Blum \& Porter, 1973). A series of experiments on cognitive "reverberation" (Blum, Geiwitz, \& Hauenstein, 1967; Blum, Hauenstein, \& Graef, 1968) utilized the insertion of 5 -sec periods of varying degrees of cognitive arousal in the 20 -sec counting interval between oral stimulus presentation of strings of six consonants and the subsequent report by the subject of the first six consonants that spontaneously come to mind. The salience of stimulus consonants in the report segment systematically varied from lowest for the -AA interpolation to highest for the +AA interpolation.

Despite the consistency of these and other findings, the use of hypnosis as a research tool inevitably engenders questions in the minds of readers unfamiliar with the approach. Methodological concerns have been addressed by Levitt and Chapman (1979) in connection with the application of hypnotic techniques to the study of emotions, psychopathology, defense mechanisms, dreams, physiological processes, and test validation. Issues specifically relevant for the validity of cognitive arousal training, such as the possible role of simulation and the operation of demand characteristics, have been discussed by Blum (1979).

The present study explores the possibility that posthypnotically controlled degrees of cognitive arousal may have distinctive EEG correlates that can serve as an independent source of verification for the method. Analysis of the amount of electrical activity at particular frequencies of the EEG may reasonably be expected to provide such evidence. Meditative states, for example, produce characteristic increases in the amplitude of 
activity in the alpha (8- to 13-Hz) EEG band (Anand, Chhina, \& Singh, 1961; Wallace, 1970). These states typically involve physical relaxation, a relative decrease in cognition, and passive focused attention. Hirai (1974), in studying stages of EEG correlates of Zen meditation in Buddhist monks, found that alpha waves first appeared at the beginning of meditation, increased in amplitude in the second stage, and decreased in frequency in the third stage. The fourth stage, accomplished only by the most experienced monks, was characterized by highvoltage rhythmical theta (4- to $7-\mathrm{Hz}$ ) waves. Sleep onset (Foulkes \& Vogel, 1965) and the stages of nocturnal sleep (Dement \& Kleitman, 1957) have long been known to have characteristic EEG patterns. Attempts to define EEG correlates of hypnosis, on the other hand, have yielded uncertain results (Sarbin \& Slagle, 1979). This is not surprising if one recognizes that brain activity and arousal levels can vary under hypnosis, depending upon instructions, just as they do in the waking state.

\section{METHOD}

\section{Selection and Hypnotic Programming of Participants}

The participants were seven undergraduate women, initially selected for their high scores (10-12) on the Stanford Hypnotic Susceptibility Scale, Form A (Weitzenhoffer \& Hilgard, 1962), as well as for their proficiency on a series of advanced hypnotic tasks particularly relevant to our experiments. Paid by the hour, they were each scheduled for 2 sessions/week over periods of 1-2 years. The early sessions were devoted to general training and practice in hypnotic skills, followed by programming procedures for specific experimental purposes, including control over degrees of cognitive arousal. The five cognitive arousal cues were practiced first under hypnosis and then posthypnotically with amnesia for the prior hypnotic programming. The average time spent on the cognitive arousal training and on subsequent checks for its efficacy was $3-4 \mathrm{~h}$.

\section{Experimental Procedure and Design}

The participant reclined on a couch in a physically relaxed position in a dimly lit experimental room. The task consisted of visualizing various objects in the room with eyes closed for 1-min periods. This visualization was done to control mental contents during the application of the cognitive arousal cues. During each 1-min visualization, one of the five cognitive arousal cues or a "baseline" instruction to remain in a normally alert waking state was given. Three different objects, chosen by the subject from among such objects as a red light, projection screen, table, chair, wall plug, and so forth, were each visualized under all six conditions, for a total of 181 -min trials. The order of conditions was random for each visualized object. A trial consisted of the following sequence: The experimenter said either "Baseline" or one of the cognitive arousal cues (-AA, $-\mathrm{A}, 0,+\mathrm{A},+\mathrm{AA})$ and waited approximately 5-10 sec for the subject to reply "Now" (a signal hypnotically prearranged to indicate, without conscious awareness of the meaning of the reply, that the subject had reached the appropriate degree of arousal). The experimenter then said "Begin," at which time the subject began the 1-min visualization. After $1 \mathrm{~min}$, the experimenter said "Stop," ending the trial and the cue condition. Fifteen-second rest periods followed each trial and 30-sec intervals preceded the set of trials for each object.

\section{EEG Recording and Analysis}

Spontaneous EEGs were recorded on a Sanborn FM tape recorder, using a Beckman R-411 dynograph to amplify the signal. Monopolar recordings from the scalp above the occipital area of the left and right hemispheres were made $\left(0_{1}\right.$ and $0_{2}$ in the standard international 10-20 placement system).

Following the recording, the EEG data were played back through a signal analyzer that provides output voltages directly proportional to the power present in specific EEG frequency bands. The analyzer was set to provide a measure of the average power present in a particular band over a period of $12 \mathrm{sec}$. Theta (4- to 7-Hz), alpha (8- to $13-\mathrm{Hz})$, and beta $(14-$ to $28-\mathrm{Hz})$ bands were analyzed. Average power readings from the first four 12 -sec epochs of the 1-min trials were recorded; the last 12-sec epoch was eliminated from the analysis because of contamination by the experimenter's "stop" instruction. Thus, for each scalp location there were 12 power readings for each EEG band (four epochs by three visualized objects) in each of the six experimental conditions. Medians for each set of 12 readings were calculated. These median voltage readings for the six conditions within each subject were then transformed to $z$ scores in order to combine the data for all seven subjects. Data from right and left hemispheres were analyzed separately.

\section{RESULTS}

Systematic changes in the amount of activity (power) in particular EEG bands occurred as degrees of cognitive arousal were varied. Figure 1 depicts the mean $\mathrm{z}$ scores for alpha, beta, and theta activity at $0_{1}$ and $0_{2}$ for the five degrees of cognitive arousal and the baseline condition. It should be kept in mind that these curves reflect the relative amount of electrical activity found for each of the six conditions within a given frequency band. Most noteworthy is the fact that the peak of cognitive arousal, +AA, has the highest amplitude for alpha and beta power and the lowest amplitude for theta in both hemispheres. The combined set of six extreme points for +AA in itself clearly cannot be attributed to the operation of chance factors. The consistency of the findings for +AA among the seven individuals also renders a chance interpretation highly improbable. In the case of alpha activity, 11 of the $14 \mathrm{z}$ scores from

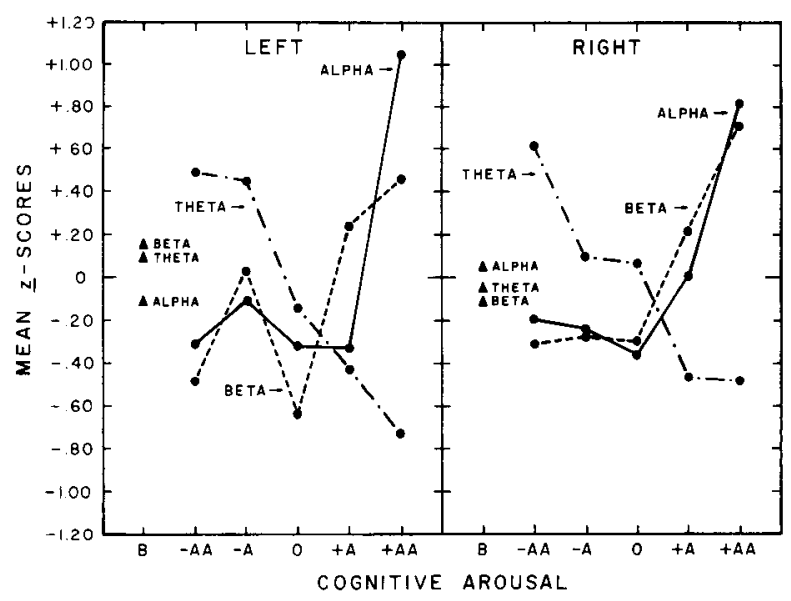

Figure 1. Mean z scores for alpha, beta, and theta activity for five degrees of cognitive arousal and a baseline condition (B). EEG recordings taken at occipital locations in left $\left(0_{1}\right)$ and right $\left(0_{2}\right)$ hemispheres. 
both hemispheres are in the positive direction, 2 are at the midpoint, and only 1 is in the negative direction. ${ }^{1}$ Similarly, for beta activity, 11 are positive and 3 are negative. The low theta mean consists of nine individual $z$ scores in the negative direction, two at the midpoint, and three in the positive direction.

In addition to the consistent set of +AA findings, Figure 1 shows a progressive decrease in theta activity from -AA to +AA for both hemispheres, again a systematic set of points that cannot be ascribed to the operation of chance. For the most part, $+A$ tends to fall somewhere between 0 and +AA (five of the six curves). In the case of alpha and beta activity, there are no observable differences among $-\mathbf{A A},-\mathbf{A}$, and 0 . The baseline $\mathrm{z}$ scores generally cluster around the midpoint of the distribution.

The data were next submitted to stepwise discriminant analysis, which also serves as a multivariate analysis of variance, providing both the multivariate statistic (Mahalanobis $\mathrm{d}^{2}$ ) and the $\mathrm{F}$ approximation to that statistic. Discriminant analysis selected four variables, alpha, theta, beta powers at $0_{1}$, and theta power at $0_{2}$. The $+A A$ condition was significantly higher than all other cued conditions for $0_{1}$ alpha $[\mathrm{F}(1,36)=10.71$, $\mathrm{p}<.005]$ and $0_{1}$ beta $[\mathrm{F}(3,34)=4.87, \mathrm{p}<.02]$ and significantly lower than all other conditions for $0_{1}$ theta $[F(2,35)=7.20, p<.005]$ and $0_{2}$ theta $[F(4,33)$ $=3.59, \mathrm{p}<.05]$. Similar trends were obtained for $+\mathrm{A}$, which was significantly higher than $-\mathrm{AA}[\mathrm{F}(3,34)=$ $4.13, \mathrm{p}<.05]$ and $-\mathrm{A}[\mathrm{F}(3,34)=3.64, \mathrm{p}<.05]$ for $0_{1}$ beta and which tended to be lower than $-\mathrm{AA}$ $[F(2,35)=3.44, p<.10]$ and $-A \quad[F(2,35)=4.70$, $\mathrm{p}<.05]$ for $0_{1}$ theta and $0_{2}$ theta $[\mathrm{F}(4,33)=3.01$, $\mathrm{p}<.10$ for $-\mathrm{AA} ; \mathrm{F}(4,33)=3.18, \mathrm{p}<.10$ for $-\mathrm{A}]$. Although only one $\mathrm{O}_{2}$ (right-hemisphere) measure was selected by the analysis, inspection of Figure 1 reveals similar trends in both hemispheres.

Inquiries conducted at the end of the session under hypnosis, when amnesia was no longer in force, lent support to the assumption that subjects responded to the posthypnotic cues as instructed. At +AA, for example, subjects reported that the image was so clear that they could "visualize tiny details," "knew exactly what the object looked like," and that it was "like looking at the object itself, not imagining it." At $+A$, subjects reported that they did not "feel as concentrated on it" as in +AA but that they "looked at it and saw the different parts." At 0 , subjects said they saw the object and "knew pretty much what it looked like." At -A, the object was hard to "visualize exactly-I tried but it never really came." At $-\mathrm{AA}$, subjects reported that they could not concentrate at all and had only vague impressions of the object ("the image was swimming and blurry"). Progressive difficulty from +AA to -AA in sustaining the image was always reported. Even at +AA, some difficulty was typically experienced near the end of the trial, and at -AA, the vague impression of the object was frequently lost and hard to bring back. The baseline condition was described as essentially similar to 0 except that concentration was lost sooner: "I thought about the object a little less." The amnesia instruction for meaning of the posthypnotic cues in the waking state was uniformly reported to have been effective. Although the amnesia instruction obviously does not "erase" the memory of prior hypnotic programming, it serves an important experimental function, in preventing conscious awareness and rumination about the cue (see Kihlstrom \& Evans, 1979, on posthypnotic amnesia).

\section{DISCUSSION}

A major outcome of the study is that posthypnotic degrees of cognitive arousal bear systematic relationships to EEG data, thereby providing independent evidence that the hypnotic programming technique yields genuine effects. Previously, validity of the cognitive arousal cues was supported inferentially from their ordered effects upon performance in diverse experimental tasks such as those mentioned in the introduction.

The particular pattern of EEG findings is most differentiated with respect to the peak of cognitive arousal, the +AA condition. Compared to the lesser degrees, +AA was accompanied by significantly larger amplitudes of alpha and beta activity and smaller amplitudes of theta activity. This pattern of results was similar in both hemispheres, although it was more marked in the left.

In relating these findings to the EEG literature, there are striking parallels to meditational states. The +AA cue, administered in this study under conditions of physical relaxation, produced an extremely alert state characterized by intense mental concentration upon a single imagined object. Many forms of meditation (e.g., transcendental meditation, yoga, and Zen) involve the focusing of attention upon a simple object, thought, or image while relaxing physically and remaining alert. As noted in the introduction, heightened alpha band activity is typically found in such states; occasionally heightened beta activity has also been observed in meditation (Banquet, 1973). The occurrence of alpha and beta in other kinds of mental tasks can only be described as inconsistent. Stigsby, Risberg, and Ingvar (1977) cited some studies in which alpha activity decreased during mental arithmetic, for example, and others in which it increased; still others have reported evidence contradictory to the widely held notion that beta activity is increased during task performance (Gevins, Zeitlin, Doyle, Schaffer, \& Callaway, 1979; Gevins, Zeitlin, Yingling, Doyle, Dedon, Schaffer, Roumasset, \& Yaeger, 1979).

Schacter (1977), reviewing the relationship between theta activity and psychological processes, concluded 
generally that "the degree to which properties of theta activity are systematically related to specific psychological processes is not yet known" (p. 47). However, he pointed out that theta has been consistently associated with the drowsy, hypnagogic state and that an important cognitive correlate of this period is a "lowered prestimulus level of alertness which is accompanied by impaired ability to process and respond to environmental information" (p. 50). On the other hand, he cited studies that suggest that increments in theta accompany selective, focused attention; in this category there are indications that persons ranked high in hypnotic susceptibility are characterized by large amounts of theta activity in various nonhypnotic conditions. The increase in theta activity found at the lower degrees of cognitive arousal in the present investigation conforms to the more common "drowsiness" interpretation.

Also compatible with the direction of the present data are findings by Kornfeld and Beatty (1977) that, as monotony increased in a continuous compensatory tracking task, $0_{1}$ theta activity increased and $0_{1}$ alpha decreased. In this connection, it is interesting to note that application of an early version of programmed arousal cues to a compensatory tracking task revealed a consistent order in number of errors from fewest at the highest degree of arousal to most at the lowest degree (Ehrlich, 1964); also, the experience of boredom was found to be strongest at - AA and decreased progressively as cognitive arousal was increased (Geiwitz, 1966).

The inconsistencies among previous EEG findings with respect to cognitive activity can perhaps be understood as a consequence of the methods used to vary level of cortical activation experimentally, for example, mental arithmetic vs. visual fixation. Because cortical activation is probably a complex function of type of task, task complexity, attention, and mental effort (e.g., Pribram, 1975), using different tasks to vary cortical activation may confound a number of variables. The present approach holds task constant while varying cognitive arousal independently. Thus, the systematic EEG correlates obtained in this study strengthen the promise of the hypnotic programming technique as a tool for exploring relationships between cognitive arousal and other facets of cognition.

\section{REFERENCES}

Anand, B. K., Сhнina, G. S., \& Singh, B. Some aspects of electroencephalographic studies in yogis. Electroencephalography and Clinical Neurophysiology, 1961, 13, 452-456.

BAnquet, J. P. Spectral analysis of EEG in meditation. Electroencephalography and Clinical Neurophysiology, 1973, 35, 143151.

BLUM, G. S. Hypnotic programming techniques in psychological experiments. In E. Fromm \& R. E. Shor (Eds.), Hypnosis: Developments in research and new perspectives. New York: Aldine, 1979.

Blum, G. S., Geiwitz, P. J., \& Hauenstein, L. S. Principles of cognitive reverberation. Behavioral Science, 1967, 12, 275288.
Blum, G. S., Geiwitz, P. J., \& Stewart, C. G. Cognitive arousal: The evolution of a model. Journal of Personality and Social Psychology, 1967, 2, 138-151.

Blum, G. S., \& Graef, J. R. The detection over time of subjects simulating hypnosis. International Journal of Clinical and Experimental Hypnosis, 1971, 19, 211-224.

Blum, G. S., Hauenstein, L. S., \& Graef, J. R. Studies in cognitive reverberation: Replications and extensions. Behavioral Science, 1968, 13, 171-177.

Blum, G. S., \& Porten, M. L. The capacity for selective concentration on color versus form of consonants. Cognitive Psychology, 1973, 5, 47-70.

Dement, W., \& Kleitman, N. Cyclic variations in EEG during sleep and their relation to eye movements, body motility, and dreaming. Electroencephalography and Clinical Neurophysiology, 1957, 9, 673-690.

EhrLich, N. J. Hypnotic control of a compensatory tracking task. Perceptual and Motor Skills, 1964, 19, 232-234.

Foulkes, D., \& Vooel, G. Mental activity at sleep onset. Journal of Abnormal Psychology, 1965, 70, 231-243.

Gerwitz, P. J. Structure of boredom. Journal of Personality and Social Psychology, 1966, 3, 592-600.

Gevins, A. S., Zeitlin, G. M., Doyle, J. C., Schaffer, R. E., \& Callaway, E. EEG patterns during "cognitive" tasks. II. Analysis of controlled tasks. Electroencephalography and Clinical Neurophysiology, 1979, 47, 704-710.

Gevins, A. S., Zeituin, G. M., Yingling, C. D., Doyle, J. C., Dedon, M. F., Schaffer, R. E., Roumasset, J. T., \& YAEGER, C. L. EEG patterns during "cognitive" tasks. I. Methodology and analysis of complex behaviors. Electroencephalography and Clinical Neurophysiology, 1979, 47, 693-703.

Hirai, T. Psychophysiology of Zen. Tokyo: Igaku Shoin, 1974.

Kinlstrom, J. F., \& Evans, F. J. Memory retrieval processes during posthypnotic amnesia. In J. F. Kihlstrom \& F. J. Evans (Eds.), Functional disorders of memory. Hillsdale, N.J: Erlbaum, 1979.

Konnfeld, C. M., \& BeAtTy, J. EEG spectra during a longterm compensatory tracking task. Bulletin of the Psychonomic Society, 1977, 10, 46-48.

LevitT, E. E., \& Chapman, R. W. Hypnosis as a research method. In E. Fromm \& R. Shor (Eds.), Hypnosis: Developments in research and new perspectives. New York: Aldine, 1979.

Pribram, K. H. Arousal, activation and effort in the control of attention. Psychological Review, 1975, 82, 116-149.

Sarbin, T. R., \& Slagle, R. W. Hypnosis and psychophysiological outcomes. In E. Fromm \& R. Shor (Eds.), Hypnosis: Developments in research and new perspectives. New York: Aldine, 1979.

Schacter, D. L. EEG theta waves and psychological phenomena: A review and analysis. Biological Psychology, 1977, 5, 47-82.

Stigsey, B., Riseera, J., \& Inavar, D. H. Electroencephalographic changes in the dominant hemisphere during memorizing and reasoning. Electroencephalography and Clinical Neurophysiology, 1977, 42, 665-675.

Wallace, R. K. Physiological effects of transcendental meditation. Science, 1970, 167, 1751-1754.

Weitzenhoffer, A. M., \& HilaARd, E. R. Stanford hypnotic susceptibility scale, Forms $A$ and B. Palo Alto, Calif: Consulting Psychologists Press, 1962.

\section{NOTE}

1. Although the binomial test cannot legitimately be applied to these directional differences because of the correlated nature of responses from the two hemispheres, the likelihood of obtaining six of seven signs in the same direction for one hemisphere on a chance basis has a $p$ value of .06 .

(Received for publication March 24, 1982; revision accepted May 18, 1982.) 